\section{Associação entre comportamentos de risco à saúde de pais e adolescentes em escolares de zona rural de um município do Sul do Brasil}

\author{
Association between health risk behaviors in \\ parents and adolescents in a rural area \\ in southern Brazil
}

\author{
1 Programa de Pós-graduação \\ em Educação Física, \\ Universidade Federal de \\ Pelotas, Pelotas, Brasil. \\ 2 Prefeitura Municipal de \\ Barão do Triunfo, Barão do \\ Triunfo, Brasil. \\ 3 Grupo de Estudos em \\ Epidemiologia da Atividade \\ Física, Universidade Federal \\ de Pelotas, Pelotas, Brasil. \\ Correspondência \\ C. O. Raphaelli \\ Programa de Pós-graduação \\ em Educação Física, \\ Universidade Federal de \\ Pelotas. \\ Rua Luiz de Camões 625, \\ Pelotas, RS 96055-630, Brasil. \\ chirleraphaelli@hotmail.com
}

\section{Abstract}

This article aimed to assess the association between health risk behaviors in parents and adolescents in a rural area. The sample included 377 schoolchildren 10 to 18 years of age and 338 parents. The following variables were analyzed: level of leisure-time and commuting physical activity, inadequate eating habits, overweight/obesity, smoking, and alcohol consumption. Prevalence rates for smoking and alcohol consumption by adolescents in the previous 30 days were $6.7 \%$ and 27\%, respectively. Forty-five percent of adolescents failed to reach the target physical activity score. Adherence to steps to healthy eating was low. Physical activity in adolescents was positively associated with that of parents, and adolescent drinking was associated with maternal alcohol consumption. There was no significant association for other behaviors. In the analysis stratified by gender, level of physical exercise in female adolescents was associated with that of fathers. $\mathrm{Pa}$ rental health risk behaviors were associated with the behaviors in the adolescent children in this rural area.

Adolescent; Behavior; Leisure Activities; Parents; Rural Population
Chirle de Oliveira Raphaelli 1,2

Mario Renato Azevedo 1,3

Pedro C. Hallal 1,3

\section{Introdução}

Mudanças no estilo de vida da população, advindas do processo de urbanização, industrialização e desenvolvimento tecnológico ocorrido nas últimas décadas, contribuem para o crescimento das doenças e agravos não transmissíveis (DANT) 1,2 . É cada vez mais precoce a exposição de crianças e adolescentes a comportamentos que desencadeiam o aparecimento de fatores de risco que levam a doenças cardiovasculares, a diabetes e ao câncer ${ }^{3}$.

Existem estudos na literatura científica que mostram prevalências de fatores de risco para DANT, inclusive em adolescentes 4,5. Entre os comportamentos de risco à saúde que devem ser urgentemente modificados pelos jovens, destacam-se os relacionados ao estilo de vida, como altos níveis de inatividade física, hábitos alimentares inadequados, tabagismo, consumo abusivo de álcool, além do excesso de peso ${ }^{6}$. Além disso, esses comportamentos são suscetíveis à intervenção, podendo ser modificados em qualquer fase da vida. Embora existam evidências de que quanto mais cedo começarem as intervenções, maiores serão as possibilidades de êxito 7,8 .

Por outro lado, especula-se que os pais possam influenciar os filhos na adoção de comportamentos relacionados à saúde, pois a família constitui o primeiro ambiente de aprendizagem das crianças e dos adolescentes 9 . O ambiente 
familiar agrega um conjunto de valores, crenças, conhecimentos e hábitos que vêm influenciar práticas que promovam a saúde de seus componentes, ou, ao contrário, aumentem a vulnerabilidade dos mesmos para as doenças ${ }^{10}$. Portanto, as atitudes dos pais estimulam, de maneira geral, a obtenção de comportamentos saudáveis ou não por parte de seus filhos 11 .

Estudos associam a presença de fatores de risco em adolescentes quando os pais ou familiares também se expõem aos mesmos fatores. Por exemplo, o fato de, pelo menos, um dos pais fumar está positivamente associado ao tabagismo do filho 12 . O consumo de bebidas alcoólicas pelos pais serve de modelo ao uso pelos seus filhos 13. Hábitos alimentares são passados de uma geração a outra, e a família tem um papel importante na influência da dieta dos adolescentes ${ }^{14}$. Com isso, a conscientização e o incentivo dos pais na adoção de um estilo de vida saudável podem influenciar os adolescentes a aderir a práticas regulares de atividade física 11 .

Estudos que relacionem comportamentos de risco à saúde entre pais e seus filhos são crescentes na literatura internacional 13,15,16,17. Pesquisas associam a presença de fatores de risco em adolescentes quando os pais ou familiares apresentam fatores similares 11,14. No entanto, no Brasil, são quase inexistentes estudos que relacionem a exposição a comportamentos de risco à saúde entre pais e adolescentes, principalmente em moradores de zona rural 6 .

Nesse sentido, objetiva-se avaliar a associação entre comportamento de risco à saúde entre os pais e adolescentes escolares de $5 \underline{a}$ a $8 \underline{a}$ série do Ensino Fundamental de um município do Sul do Brasil.

\section{Métodos}

Um estudo transversal de base escolar foi realizado na zona rural de Barão do Triunfo, Rio Grande do Sul, Brasil. O município, com território de $436,4 \mathrm{~km}^{2}$, tem 7.018 habitantes, sendo que 53\% da população é do sexo masculino, e $90 \%$ são moradores de zona rural. O município localizase na Região Carbonífera do Estado do Rio Grande do Sul, possui um Produto Interno Bruto per capita de R\$ 8.522 e Índice de Desenvolvimento Humano de 0,74 18,19.

Barão do Triunfo foi eleito para a pesquisa por ser caracterizado um município de zona rural, tendo, como característica, a economia baseada na agricultura (cultivo de fumo) e parte na pecuária (bovinos). Os hábitos culturais presentes no município foram trazidos por imigrantes italianos, poloneses, alemães e espanhóis 19 .
O sistema de educação é composto por 8 escolas municipais distribuídas na zona rural, 1 escola estadual e 1 instituição filantrópica de educação localizadas na zona urbana. As escolas municipais atendem desde a pré-escola até o Ensino Fundamental; a estadual atende o Ensino Médio; e a instituição filantrópica (Associação de Pais e Amigos dos Excepcionais) atende alunos portadores de deficiências. O Ensino Superior é atendido por instituições localizadas em municípios vizinhos 19 .

Apesar dos suportes educacionais oferecidos pelo município, a taxa de analfabetismo de indivíduos com 15 anos ou mais é de 15,87\% e de $30,4 \%$ para analfabetos funcionais, ou seja, aqueles com menos de 4 anos completos de estudos 20.

Conforme o Censo Escolar de 2009, havia 913 alunos de Ensino Fundamental matriculados na Rede Municipal de Educação, e, desses, 385 eram alunos de $5 \underline{a}$ a $8 \underline{a}$ série, sendo distribuídos nas escolas com Ensino Fundamental completo 21.

A amostra incluiu os adolescentes matriculados, entre a $5 \underline{a}$ e a $8 \underline{a}$ séries, nas três escolas da Rede Municipal de Educação e seus pais (biológicos ou não). As escolas foram selecionadas para coleta de dados devido à presença de Ensino Fundamental completo (5a e a 8a série), sendo que todas estão localizadas na zona rural do município.

A coleta de dados foi realizada entre março e setembro de 2010, tendo duas etapas. A primeira, com os escolares, foi realizada em sala de aula. Foram realizadas, no mínimo, três visitas em sala de aula para cada turma, para minimizar as perdas. Num segundo momento, a coleta de dados com os pais foi realizada em reunião agendada com os mesmos. Caso esses não comparecessem na escola era remarcada outra data, e, após, realizada uma visita no domicílio.

Foram excluídos do estudo indivíduos com incapacidade mental de responder ao questionário, adolescentes que não estavam presentes em nenhuma das visitas nas salas de aula e adultos que não compareceram às reuniões ou não estavam no domicílio no momento da visitação para a coleta de dados.

Para a coleta, foram construídos dois questionários, um para os adultos e outro para os adolescentes, os quais foram previamente testados no piloto realizado em um município vizinho com amostra similar, com a finalidade de adequar os instrumentos de coleta de dados. Os instrumentos, autoaplicáveis, foram entregues aos escolares em sala de aula sem a presença dos professores e aos pais em uma sala previamente organizada. Os questionários foram respondidos individualmente depois de serem dadas as devidas instru- 
ções, sendo os mesmos revisados, posteriormente, pela coordenadora do estudo. O tempo de aplicação foi de 40 minutos (30-60 minutos). Em seguida, em uma sala preparada para tal, foram realizadas as medidas antropométricas. A fase de aplicação do questionário foi realizada por uma nutricionista, e as medidas, por três professores de educação física, previamente treinados para a realização das medidas. O controle de qualidade foi realizado em $10 \%$ das medidas antropométricas com os adolescentes para a correção dos erros e conferência dos dados coletados, garantindo, assim, a validade interna do estudo.

Medidas de massa corporal foram realizadas em balança digital da marca Plenna (capacidade de 150kg e precisão de 100g - São Paulo, Brasil), e medidas de estatura, em estadiômetro tipo portátil da marca Sanny (medida até $210 \mathrm{~cm}$ e precisão de $1 \mathrm{~mm}$ - São Bernardo do Campo, Brasil), previamente e periodicamente calibrados. Para realização das medidas, os adolescentes e os adultos deveriam estar com roupas leves, sem calçados e em posição ortostática 22 . Cada medida foi aferida duplamente para posterior realização da média e obtenção de um resultado preciso.

Também foram obtidas informações dos comportamentos de risco à saúde - ser ativo (sim/não), hábitos alimentares inadequados, uso de cigarros e consumo de bebidas alcoólicas, sobrepeso/obesidade - de adolescentes e seus pais. Além disso, foram obtidas variáveis socioeconômicas dos pais e demográficas e antropométricas dos pais e dos adolescentes. As variáveis independentes analisadas foram sexo e idade dos adolescentes e escolaridade dos pais (em anos completos de estudo).

O nível de atividade física de adolescentes foi avaliado com o instrumento de Bastos et al. 23, o qual investiga as atividades realizadas nos últimos sete dias anteriores à aplicação do questionário, excluindo as atividades realizadas durante as aulas de educação física escolar. Foram coletadas informações de frequência e duração das atividades físicas realizadas no período de lazer e como deslocamento. Após, foram considerados como ativos os jovens que acumulavam, pelo menos, 300 minutos por semana de atividades físicas 24 .

Em relação à atividade física dos pais, foi aplicada a versão longa do Questionário Internacional de Atividades Físicas (IPAQ) 25. Para fins de análise, foram consideradas apenas as seções do IPAQ referentes à atividade física de lazer e ao deslocamento 26 . Um escore semanal de atividade física foi elaborado, multiplicando a frequência semanal (dias) pela duração média (minutos) das atividades físicas moderadas e vigorosas, sendo que o tempo semanal despendido em ati- vidades de intensidade vigorosa foi multiplicado por dois, conforme sugerido em publicações anteriores ${ }^{27}$. Indivíduos com escore de atividade física igual ou superior a 150 minutos por semana foram considerados ativos 28 .

Informações sobre hábito alimentar de adolescentes e de seus pais foram obtidas a partir de um questionário elaborado, baseado nos Dez Passos para uma Alimentação Saudável do Ministério da Saúde 29,30. As questões desse instrumento permitiram verificar a frequência de consumo dos alimentos no período de um ano anterior à entrevista. Para cada passo, utilizou-se uma variável dicotômica (adesão sim/não). A exposição ao comportamento de risco à saúde, hábitos alimentares inadequados tanto para adultos quanto para adolescentes, foi considerada a não adesão a cada passo, com base nos critérios descritos na Tabela 1.

O passo 10 não foi categorizado neste estudo, pois esse passo se refere ao hábito de atividade física, tabagismo, ingestão de bebidas alcoólicas e estado nutricional, os quais foram analisados de forma mais detalhada pelos instrumentos aqui descritos.

Para a avaliação dos comportamentos de risco à saúde, uso de cigarros e consumo de bebidas alcoólicas, foram utilizadas as questões propostas pelo Centro Brasileiro de Informações Sobre Drogas Psicotrópicas ${ }^{31}$ para o questionário de adultos e adolescentes. O consumo de álcool foi considerado positivo quando os respondentes referiam ter consumido qualquer quantidade de bebidas alcoólicas no último mês, e o fumo foi considerado positivo quando os respondentes referiam ter usado qualquer quantidade de fumo nos últimos 30 dias.

O excesso de peso (sobrepeso e obesidade), definido como comportamento de risco à saúde, foi classificado a partir do índice de massa corporal (IMC). O IMC dos jovens foi classificado em percentil, segundo idade e sexo ${ }^{32}$. Para categorizar os pontos de corte do IMC dos pais, recorreu-se à classificação indicada pela Organização Mundial da Saúde (normal: $<25 \mathrm{~kg} / \mathrm{m}^{2}$; sobrepeso: $25-29,9 \mathrm{~kg} / \mathrm{m}^{2}$; obesidade: $\left.\geq 30 \mathrm{~kg} / \mathrm{m}^{2}\right) 33$.

O software Epi Info 6.0 (Centers for Disease Control and Prevention, Atlanta, Estados Unidos) foi utilizado para dupla digitação dos dados. Nesse programa, foi possível utilizar o item Validate e corrigir os erros entre os bancos. A análise dos dados foi realizada no Stata 11.0 (Stata Corp., College Station, Estados Unidos), e foram utilizados os recursos da estatística descritiva: média, desvio-padrão (DP), frequência relativa e intervalo de $95 \%$ de confiança (IC95\%), seguidos de análise bivariada usando qui-quadrado e análise ajustada por 
Critérios utilizados para adesão aos 10 Passos para uma Alimentação Saudável.

\begin{tabular}{ll}
\hline Passos & \multicolumn{1}{c}{ Adesão ao passo: positiva } \\
\hline 1 & Realização de cinco ou mais refeições ao dia \\
2 & Consumo de seis ou mais porções de cereais, raízes ou tubérculos ao dia \\
3 & Consumo de três ou mais porções de frutas ao dia e três ou mais porções de legumes ou verduras ao dia \\
4 & Consumo de arroz e feijão combinados cinco ou mais vezes por semana \\
5 & Consumo de leite e derivados três vezes ou mais por dia, consumo de carnes, peixes ou ovos uma vez por \\
6 & Consumo de óleos e gorduras uma vez ou menos por dia, utilização de manteiga, margarina ou óleo nas \\
7 & Consumo de refrigerantes duas vezes ou menos por semana, consumo de sucos industrializados duas vezes \\
& cou menos por semana, consumo de bolos, bolachas doces ou recheadas duas vezes ou menos por semana, \\
& Consumo de doces, balas, chocolates e guloseimas duas vezes ou menos por semana
\end{tabular}

regressão de Poisson com variância robusta 34 . Em todas as análises, utilizou-se um nível de 5\% de significância.

O estudo foi aprovado pelo Comitê de Ética em Pesquisa com seres humanos da Escola Superior de Educação Física, da Universidade Federal de Pelotas, sob o protocolo $n^{\circ}$. 068/2009. Os participantes da pesquisa (pais ou responsáveis dos adolescentes) assinaram o termo de consentimento livre e esclarecido. Em todas as fases da pesquisa, foram garantidos o anonimato, a liberdade de recusa e a possibilidade de retirada do consentimento.

\section{Resultados}

Dos 412 escolares elegíveis, 377 participaram do estudo, perfazendo um total de $8,5 \%$ de perdas e recusas. Dos 725 pais e mães, 338 participaram do estudo, perfazendo uma taxa de $46,6 \%$ de participação. Entre os escolares respondentes à pesquisa, $27,7 \%$ desses estavam matriculados

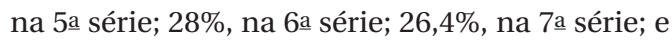
$17,9 \%$, na 8 a série. A idade média dos adolescentes era de 12,9 (DP $\pm 1,8)$, variando de 10-18 anos. Percebeu-se que $18,93 \%$ dos escolares estavam acima de 14 anos; desses, 23,62\% eram meninos, e $13,64 \%$, meninas.

A Tabela 2 apresenta as variáveis demográficas, socioeconômicas e de comportamentos de risco à saúde dos adolescentes e de seus pais. A amostra de adolescentes foi predominantemen- te composta por rapazes $(53,1 \%)$. O comportamento de risco, fumo nos últimos 30 dias, foi relatado por $6,7 \%$ dos adolescentes, $46,5 \%$ dos pais e $13,6 \%$ das mães. O consumo de bebidas alcoólicas no último mês foi referido por $27 \%$ dos escolares, $46,4 \%$ dos pais e $26,7 \%$ das mães. Com relação ao estado nutricional, $24,8 \%$ dos adolescentes, $62,7 \%$ dos pais e $66,6 \%$ das mães apresentaram excesso de peso. Do total, $45 \%$ dos jovens, $59,8 \%$ dos pais e $75 \%$ das mães não atingiram o escore de atividade física. A frequência de adesão aos passos para uma alimentação saudável ficou baixa em todos os passos, exceto no referente ao consumo semanal de arroz e feijão combinados (passo 4), o qual atingiu 82,3\% de adesão pelos adolescentes, $87,5 \%$ dos pais e $85,8 \%$ das mães. Os hábitos alimentares inadequados são demonstrados pela baixa adesão dos adolescentes ao passo $1(31,4 \%)$, passo $2(31,5 \%)$ e passo $9(31,8 \%)$ e uma adesão extremamente baixa aos passos 3 (consumo de frutas e legumes), 5 (consumo de leite e carnes e hábito de retirar a gordura aparente dessas), 6 (consumo de óleos e gorduras), 7 (consumo de refrigerantes e guloseimas) e 8 (consumo de industrializados).

A Tabela 3 mostra a análise bruta das variáveis de comportamento de risco à saúde de adolescentes conforme as variáveis de comportamento dos pais. O tabagismo dos pais não se mostrou associado ao fumo dos adolescentes no último mês. Apesar de a prevalência de fumo ser mais elevada nos filhos de pais fumantes, o resultado não foi significativo $(p=0,32)$. O consumo de 
Descrição da amostra de adolescentes e seus pais conforme variáveis demográficas, socioeconômicas e de comportamentos de risco à saúde. Barão do Triunfo, Rio Grande do Sul, Brasil.

\begin{tabular}{|c|c|c|c|}
\hline Variável & Adolescentes $(n=377)$ & Pais $(n=165)$ & Mães $(n=173)$ \\
\hline \multicolumn{4}{|l|}{ Sexo } \\
\hline Masculino & $53,1 \%$ & & \\
\hline Feminino & $46,9 \%$ & & \\
\hline Idade em anos completos (média \pm DP) & $12,9 \pm 1,8$ & $45,2 \pm 8,7$ & $40,8 \pm 8,1$ \\
\hline Escolaridade (média \pm DP) & & $12,5 \pm 26,5$ & $8,1 \pm 16,1$ \\
\hline \multicolumn{4}{|l|}{ Fumo nos últimos 30 dias } \\
\hline Não & $93,4 \%$ & $53,5 \%$ & $86,4 \%$ \\
\hline $\operatorname{Sim}$ & $6,7 \%$ & $46,5 \%$ & $13,6 \%$ \\
\hline \multicolumn{4}{|l|}{ Consumo de álcool nos últimos 30 dias } \\
\hline Não & $73,0 \%$ & $53,6 \%$ & $73,3 \%$ \\
\hline $\operatorname{Sim}$ & $27,0 \%$ & $46,4 \%$ & $26,7 \%$ \\
\hline \multicolumn{4}{|l|}{ IMC * } \\
\hline Normal & $75,2 \%$ & $37,4 \%$ & $33,3 \%$ \\
\hline Sobrepeso & $10,7 \%$ & $49,4 \%$ & $43,1 \%$ \\
\hline Obesidade & $14,1 \%$ & $13,3 \%$ & $23,5 \%$ \\
\hline \multicolumn{4}{|l|}{ Atividade física ** } \\
\hline Ativo: não & $45,0 \%$ & $59,8 \%$ & $75,0 \%$ \\
\hline Ativo: sim & $55,0 \%$ & $40,2 \%$ & $25,0 \%$ \\
\hline \multicolumn{4}{|l|}{ Passos da alimentação saudável } \\
\hline Adesão ao passo 1 & $31,4 \%$ & $6,9 \%$ & $8,8 \%$ \\
\hline Adesão ao passo 2 & $31,5 \%$ & $14,7 \%$ & $13,0 \%$ \\
\hline Adesão ao passo 3 & $5,6 \%$ & $0,0 \%$ & $0,0 \%$ \\
\hline Adesão ao passo 4 & $82,3 \%$ & $87,5 \%$ & $85,8 \%$ \\
\hline Adesão ao passo 5 & $0,6 \%$ & $0,0 \%$ & $0,0 \%$ \\
\hline Adesão ao passo 6 & $4,8 \%$ & $6,4 \%$ & $7,4 \%$ \\
\hline Adesão ao passo 7 & $14,8 \%$ & $34,8 \%$ & $36,5 \%$ \\
\hline Adesão ao passo 8 & $0,9 \%$ & $0,8 \%$ & $0,7 \%$ \\
\hline Adesão ao passo 9 & $31,8 \%$ & $24,0 \%$ & $16,7 \%$ \\
\hline
\end{tabular}

DP: desvio padrão; IMC: índice de massa corporal.

* Ponto de corte utilizado para IMC de adolescente 32 e adulto 33;

** Ponto de corte utilizado para nível de atividade física de adolescente 24 e adulto 28 .

bebida alcoólica dos adolescentes esteve associado ao consumo de álcool das mães. Houve associação entre a presença de excesso em escolares e suas mães. $\mathrm{O}$ adolescente ser ativo fisicamente esteve associado à atividade física dos pais. O hábito alimentar dos adolescentes não se mostrou associado com o comportamento de risco dos pais.

Após análise ajustada por sexo, idade do adolescente e escolaridade dos pais (Tabela 4), o consumo de álcool de escolares e suas mães, assim como atividade física do pai e do filho mantiveram uma associação positiva. O consumo maior ou igual a seis vezes ao dia de cereais, raízes ou tubérculos (passo 2) de mães mostrou- se associado ao mesmo padrão de consumo dos escolares ( $p=0,02)$.

Os pais não terem o comportamento de risco à saúde, ou seja, serem ativos fisicamente, mostrou associação com o nível de atividade física de moças e rapazes, representado pela Figura 1. $\mathrm{O}$ pai ser ativo mostrou-se associado à atividade física das meninas ( $p=0,02$ ), não tendo significância estatística na atividade física dos meninos. A mãe ser ativa não mostrou associação na atividade física de meninos e meninas. 
Comportamento de risco à saúde de adolescentes conforme o comportamento dos pais: análise bruta. Barão do Triunfo, Rio Grande do Sul, Brasil.

\begin{tabular}{lcccccc}
\hline Variáveis & \multicolumn{3}{c}{ Pais } & \multicolumn{3}{c}{ Mães } \\
& Não (\%) & Sim (\%) & Valor de $\mathbf{p}$ * & Não & Sim & Valor de $\mathbf{p}$ * \\
\hline Fumo nos últimos 30 dias & 2,8 & 6,4 & 0,32 & 3,7 & 0,0 & 0,38 \\
Consumo de álcool nos últimos 30 dias & 21,3 & 27,3 & 0,40 & 19,0 & 34,2 & 0,05 \\
Sobrepeso/Obesidade & 19,4 & 25,5 & 0,52 & 14,7 & 32,8 & 0,05 \\
Ativo & 48,5 & 66,7 & 0,02 & 53,9 & 65,1 & 0,20 \\
Adesão ao Passo 1 & 29,9 & 27,3 & 0,85 & 26,6 & 40,0 & 0,27 \\
Adesão ao Passo 2 & 29,0 & 38,9 & 0,40 & 29,7 & 52,9 & 0,06 \\
Adesão ao Passo 4 & 82,4 & 82,1 & 0,98 & 81,0 & 81,8 & 0,92 \\
Adesão ao Passo 6 & 6,7 & 0,0 & 0,51 & 3,0 & 12,5 & 0,17 \\
Adesão ao Passo 7 & 10,0 & 13,3 & 0,57 & 9,4 & 18,4 & 0,13 \\
Adesão ao Passo 9 & 30,8 & 34,3 & 0,70 & 32,8 & 38,5 & 0,58 \\
\hline
\end{tabular}

* Teste do qui-quadrado para heterogeneidade.

Comportamento de risco à saúde de adolescentes conforme o comportamento dos pais: análise ajustada. Barão do Triunfo, Rio Grande do Sul, Brasil.

\begin{tabular}{|c|c|c|c|c|}
\hline \multirow[t]{2}{*}{ Desfechos } & \multicolumn{2}{|c|}{ Pai } & \multicolumn{2}{|c|}{ Mãe } \\
\hline & $\mathrm{RP}(\mathrm{IC} 95 \%)$ * & Valor de $\mathrm{p}$ & $\mathrm{RP}(\mathrm{IC} 95 \%)$ * & Valor de $p$ \\
\hline Fumo nos últimos 30 dias & $2,3(0,4 ; 12,3)$ & 0,34 & $\star \star$ & $\star \star$ \\
\hline Consumo de álcool nos últimos 30 dias & $1,2(0,7 ; 2,2)$ & 0,48 & $1,8(1,0 ; 3,1)$ & 0,05 \\
\hline Sobrepeso/obesidade & $1,3(0,5 ; 3,0)$ & 0,60 & $2,1(0,9 ; 5,2)$ & 0,09 \\
\hline Ativo & $1,4(1,1 ; 1,8)$ & 0,02 & $1,1(0,9 ; 1,5)$ & 0,37 \\
\hline Adesão ao passo 1 & $1,0(0,4 ; 2,8)$ & 0,99 & $1,5(0,8 ; 3,0)$ & 0,22 \\
\hline Adesão ao passo 2 & $1,3(0,6 ; 2,5)$ & 0,50 & $1,9(1,1 ; 3,4)$ & 0,02 \\
\hline Adesão ao passo 4 & $1,0(0,8 ; 1,3)$ & 0,97 & $1,0(0,8 ; 1,2)$ & 0,83 \\
\hline Adesão ao passo 6 & $\star \star$ & $\star \star$ & $4,8(0,6 ; 37,9)$ & 0,14 \\
\hline Adesão ao passo 7 & $1,9(0,6 ; 5,7)$ & 0,27 & $1,9(0,8 ; 4,6)$ & 0,14 \\
\hline Adesão ao passo 9 & $1,0(0,6 ; 1,7)$ & 0,94 & $1,2(0,7 ; 2,0)$ & 0,54 \\
\hline
\end{tabular}

IC95\%: intervalo de 95\% de confiança; RP: razão de prevalência.

* Ajustada por sexo e idade do adolescente e escolaridade dos pais;

** Não é possível estimar a razão de prevalências, visto que o valor de uma das caselas é 0 .

\section{Discussão}

O presente estudo avaliou a associação de comportamentos de risco à saúde entre os pais e adolescentes em um município de pequeno porte. Algumas limitações deste devem ser consideradas. Por ser um estudo transversal, a coleta dos desfechos e das variáveis de exposição se deu em um único momento no tempo, o que impede a avaliação de temporalidade nas associações en- contradas. Em função de o tamanho amostral ser pequeno, não foi possível estratificar as análises por sexo e idade dos escolares, nos impedindo de explorar, mais detalhadamente, o efeito das exposições sobre os desfechos segundo essas variáveis. Tal preocupação se justifica pelas diferenças quanto à susceptibilidade de adolescentes aos comportamentos de risco entre meninos e meninas, assim como adolescentes de diferentes idades. Outra limitação refere-se ao fato de que a 


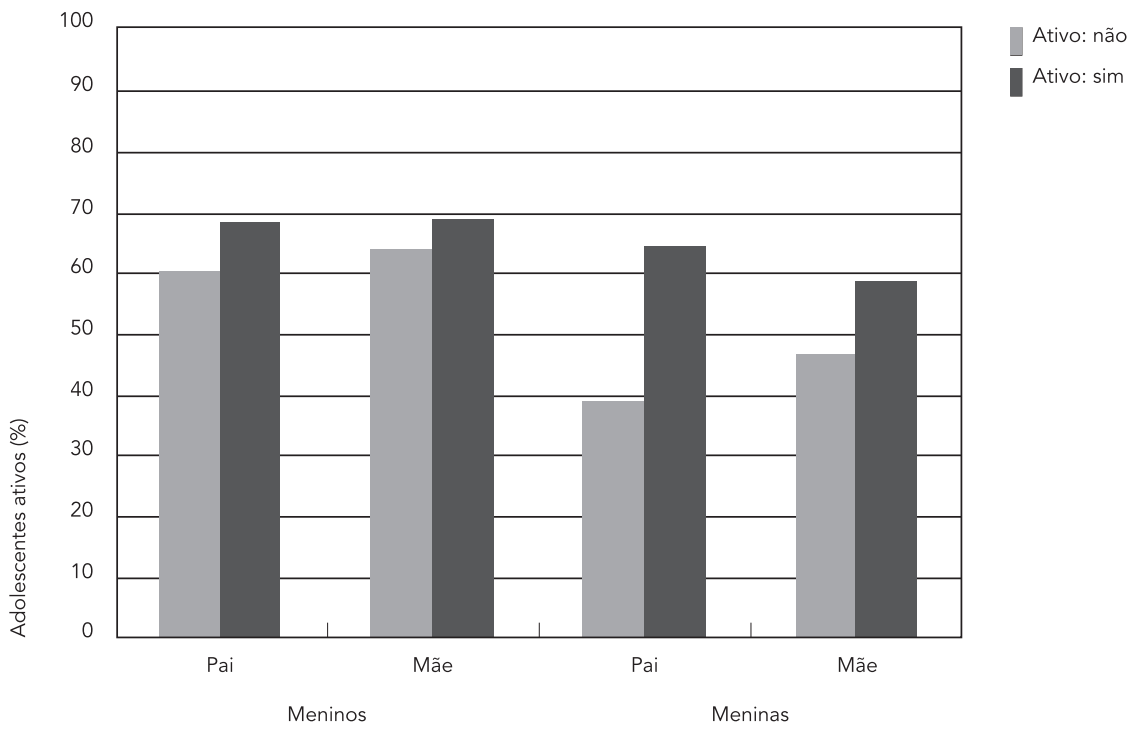

coleta de dados de consumo alimentar baseouse apenas na frequência de consumo, sem analisar a quantidade de alimentos.

O baixo percentual de respostas de pais e mães juntos também representou uma importante limitação. Quando os pais e mães foram convidados a participar do estudo, houve uma tendência de que somente um deles aceitasse o convite em cada família, o que fez com que tenhamos dados de um dos pais para a maioria dos adolescentes (68\%). A consequência desse baixo percentual de participação é o baixo poder para algumas análises. Por exemplo, até razões de prevalência superiores a 2,0 não alcançaram significância estatística quando o desfecho ou a exposição possuíam baixa prevalência. Em outras análises, não foi possível estimar razões de prevalência pelo fato de uma das caselas ter valor 0 . Além disso, supõe-se que os pais não respondentes possam ter menor grau de escolaridade, o que também é um indicador importante dessa população em vista do alto percentual de analfabetos. Finalmente, é conhecido que os dados do atual estudo foram coletados no Município de Barão do Triunfo, e isso sugere cautela na generalização dos resultados para a população em geral.

A prevalência de tabagismo autorrelatado pelos adolescentes mostrou-se similar a de estudos realizados com adolescentes de zona ur- bana 35,36 ou rural 37. Estudo realizado em área urbana e rural mostrou que o tabagismo dos pais esteve fortemente associado ao uso de cigarros pelos filhos 38 , um achado também encontrado em várias outras pesquisas 15,36,39,40, embora não todas 41,42. No presente estudo, as associações foram no sentido esperado, mas não atingiram significância estatística devido ao baixo poder, especialmente nas análises incluindo as mães, as quais apresentaram uma prevalência menor de tabagismo se comparadas aos pais. Previa-se que a prevalência de exposição a esse comportamento de risco à saúde fosse mais elevada, pois o município tem economia basicamente agrícola advinda da produção de fumo. Por outro lado, é plausível pensarmos na efetividade das medidas regulatórias e na proibição da propaganda de cigarros já instituídas pelo governo brasileiro há alguns anos.

O comportamento de consumo de bebidas alcoólicas no último mês foi referido por $27 \%$ dos escolares. Esses dados se mostraram semelhantes aos encontrados em outros estudos 36,43,44. Dentre os adolescentes, a prevalência de consumo de bebida alcoólica no último mês era maior quando sua mãe mostrava o mesmo comportamento de risco à saúde, um achado que é apoiado por estudos prévios 13,36,45. Tem sido descrito que o estímulo ao uso da bebida alcoólica pelo 
adolescente é incentivado a partir dos próprios parentes 46 , também pelo histórico de uso de álcool na família 13,47 , pela facilidade de acesso à bebida dentro de casa 48,49 ou ainda pela falta de apoio afetivo familiar ao adolescente ${ }^{50}$. Além disso, a população estudada é residente em município de pequeno porte, e os lazeres ofertados nesse local foram trazidos por descendentes de origem italiana (vinho) e alemã (cerveja) e estão arraigados à cultura 51. Com isso, é comum o consumo de bebidas alcoólicas em festas populares, encontros de clubes e associações e na própria residência 51 .

O sobrepeso e a obesidade continuam tendo um interesse especial para a saúde pública 52 . Menores prevalências de excesso de peso em adolescentes moradores de zona rural comparados com os de zona urbana são demonstradas na literatura 53,54, mas nosso estudo encontrou valores superiores a estudos nacionais prévios 55,56 , incluindo um realizado em zona rural 57 . O estado nutricional dos pais não mostrou associação com a prevalência de sobrepeso e obesidade nos escolares, enquanto o estado nutricional das mães relacionou-se com tal desfecho. A literatura demonstra correlação entre o IMC dos pais e dos filhos 58 , associando o ganho de peso na infância e adolescência aos comportamentos inadequados da alimentação de seus pais 59 .

A exposição ao comportamento de risco à saúde, hábitos inadequados de alimentação, ficou demonstrada pela baixa frequência de adesão aos passos da alimentação saudável. Comparando com a literatura, a adesão ao passo 1 pelos adolescentes foi superior ao observado em outra investigação ${ }^{60}$. A baixa adesão aos passos 2 e 3 está de acordo com estudos prévios 61,62, fato preocupante em virtude de o consumo de frutas e hortaliças e alimentos integrais auxiliar na prevenção de DANT 63. O passo 4 foi o que teve a maior frequência de adesão, a qual se mostrou bastante similar com algumas investigações 30,64 , discordando de outra 65 . O prato que mais caracteriza o nosso hábito alimentar diário é o feijão com arroz, e esse satisfaz as necessidades básicas do organismo com relação às calorias e proteínas 51 . A adesão ao passo 6 é baixa, o que demonstra que muitos adolescentes têm um consumo acima do preconizado de óleos e gorduras 61. Achados da literatura 62,64 também mostraram alto consumo de bebidas doces, guloseimas e alimentos industrializados entre adolescentes. Os dados dos passos 8 e 9 corroboram com a literatura 17 .

A adesão aos passos para uma alimentação saudável mostrou-se, em geral, baixa tanto para adolescentes quanto para os pais. Presumese que a compra de alimentos saudáveis, como frutas e verduras, pela população estudada, seja difícil devido à dificuldade de acesso aos supermercados centrais e à monocultura utilizada pelos agricultores locais, os quais não têm o hábito de produzir frutas e verduras. Outra justificativa para os inadequados hábitos alimentares da população é mostrada pela diversidade cultural da alimentação, advinda de várias etnias ${ }^{19}$. Na alimentação de descendentes italianos, é comum perceber a existência de refeições abundantes, com excesso de consumo de cereais (massas e polentas) e de gorduras (queijos), e a alimentação de descendentes alemães, poloneses e portugueses apresenta maior consumo de cereais (batatas, centeio), de alimentos embutidos, defumados e salgados, além da carne suína 19,51.

Ressalta-se o papel determinante da família na formação dos hábitos alimentares saudáveis do adolescente 17,66,67. Mas, no presente estudo, ao avaliar a associação entre os hábitos alimentares dos adolescentes e seus pais, não houve associação entre a adesão à maioria dos passos da alimentação saudável pelos pais e adolescentes. Apenas o segundo passo, relativo ao consumo de cereais, raízes ou tubérculos, mostrou-se associado entre adolescentes e suas mães. Esse resultado sugere que as mães detêm a organização da alimentação no ambiente familiar.

A prática de atividade física entre os adolescentes mostrou frequência superior a de estudos nacionais de zona urbana 25,30,68,69. Investigações trazem resultados divergentes ao comparar nível de atividade física e área de residência. Enquanto um estudo mostrou que adolescentes residentes em áreas rurais apresentam menos chances de serem suficientemente ativos 70 , outro estudo mostrou uma prevalência de comportamento sedentário menor entre os residentes de áreas rurais 71 . No presente estudo, a prevalência do comportamento de risco à saúde, inatividade física, foi considerada elevada. Pressupõe-se que esse fato deve-se à falta de ambiente físico adequado para a realização das práticas desportivas, à inexistência de ginásios e quadras esportivas na área rural do município, além da grande utilização de transporte escolar, como deslocamento, pelos adolescentes.

Ao associar a prática de atividade física dos pais e do adolescente, fica evidente o modelo familiar. Essa relação mostrou associação entre a atividade física do pai e do adolescente na análise bruta. Na ajustada, a chance de o adolescente ser ativo ficou em $40 \%$ quando seu pai também o é. Ao estratificar a análise por sexo do escolar, o pai ser ativo esteve associado à maior chance de os adolescentes do sexo feminino realizarem atividade física. Outros estudos verificaram uma associação positiva e significativa entre a prática 
desportiva dos pais e de seus filhos 1,72, além do apoio materno, suportando maior risco da realização da atividade física entre adolescentes 73 . Contrariando nossos resultados, um estudo longitudinal com jovens de 7-12 anos não suportou a hipótese que a atividade física, no tempo livre dos adolescentes, estaria associada à atividade física de seus pais 74 .

A hipótese central testada no presente estudo é de que o comportamento de risco à saúde dos pais influencia a exposição ao mesmo comportamento pelos adolescentes. Tal questão científica é relevante no contexto das áreas rurais, para as quais a disponibilidade de dados é bastante limitada. Tendo em vista a direção e a magnitude das associações aqui apresentadas, é possível con- cluir que tal hipótese é plausível, embora várias associações não tenham alcançado significância estatística devido ao baixo poder relacionado ao baixo percentual de participação dos pais. A adoção de um estilo de vida saudável deve ser estimulada na infância e na adolescência, pois, nessas fases, são formados os hábitos alimentares e de atividade física 67 , e pode ser prevenida a experimentação de bebidas alcoólicas e de cigarros. Com os dados do presente estudo, podemse planejar estratégias de prevenção em médio e longo prazo e promover mudanças de comportamentos ligados ao estilo de vida de toda a família, visando à melhoria na qualidade de vida e à redução de problemas de saúde no futuro dessa população.

\section{Resumo}

Objetivou-se avaliar a associação entre comportamentos de risco à saúde dos pais e adolescentes escolares de zona rural. Participaram 377 escolares entre 10 e 18 anos e 338 pais. Foram analisados: nível de atividade física no lazer e deslocamento, hábitos de alimentação inadequados, excesso de peso, uso de cigarros e consumo de bebidas alcoólicas. A prevalência de relato de uso de cigarro e álcool nos últimos 30 dias entre os jovens foi de 6,7\% e 27\%, respectivamente. Quarenta e cinco por cento dos adolescentes não atingiram o escore de atividade física. A adesão aos passos para uma alimentação saudável foi baixa. A atividade física dos adolescentes esteve relacionada de forma positiva com a dos pais, e o consumo de álcool da mãe se associou de forma positiva com o consumo do adolescente. Para os outros comportamentos, não houve associação significativa. Na análise estratificada por sexo, o nível de atividade física do pai se associou ao nível de atividade física das meninas. O comportamento de risco à saúde dos pais associa-se ao mesmo comportamento dos adolescentes de zona rural.

Adolescente; Comportamento; Atividade de Lazer; Pais; População Rural

\section{Colaboradores}

C. O. Raphaelli trabalhou em todas as etapas da elaboração do artigo. M. R. Azevedo trabalhou na metodologia e elaboração do banco de dados, colaborou na elaboração do artigo e na revisão crítica. P. C. Hallal coordenou a análise dos dados e colaborou na revisão crítica do artigo.

\section{Agradecimentos}

À Prefeitura Municipal e à Secretaria Municipal de Educação e Cultura de Barão do Triunfo, Rio Grande do Sul, aos diretores, professores, alunos e pais que participaram da coleta de dados. 


\section{Referências}

1. Seabra AFT, Maia JA, Mendonça DM, Thomis M, Caspersen CJ, Fulton JE. Age and sex differences in physical activity of Portuguese adolescents. Med Sci Sports Exerc 2008; 40:65-70.

2. Boutayeb A, Boutayeb S. The burden of non communicable diseases in developing countries. Int $\mathrm{J}$ Equity Health 2005; 4:2.

3. World Health Organization. Inequalities young people's health: key findings from the Health Behaviour in School-aged Children (HBSC) 2005/2006 survey fact sheet. Copenhagen: World Health Organization; 2008.

4. Kelishadi R, Sadri G, Tavasoli AA, Kahbazi M, Roohafza HR, Sadeghi M, et al. Cumulative prevalence of risk factors for atherosclerotic cardiovascular diseases in Iranian adolescents. J Pediatr (Rio J.) 2005; 81:447-53.

5. Guedes DP, Guedes JE, Barbosa DS, Oliveira JA Stanganelli LC. Cardiovascular risk factors in adolescents: biological and behavioral indicators. Arq Bras Cardiol 2006; 86:439-50.

6. Farias Júnior JC, Nahas MV, Barros MVG, Loch MR, Oliveira ESA, De Bem MFL, et al. Comportamentos de risco à saúde em adolescentes no Sul do Brasil: prevalência e fatores associados. Rev Panam Salud Pública 2009; 25:344-52.

7. World Health Organization. The World Health Report 2002: reducing risk, promoting healthy life. Geneva: World Health Organization; 2002.

8. Santos MG, Pegoraro M, Sandrini F, Macuco EC. Risk factors for the development of atherosclerosis in childhood and adolescence. Arq Bras Cardiol 2008; 90:276-83.

9. Duarte MEB. Influência dos estilos de vida familiar no desenvolvimento do excesso de peso e obesidade em crianças em idade pré-escolar [Tese de Doutorado]. Lisboa: Universidade de Lisboa; 2007.

10. Instituto Brasileiro de Geografia e Estatística. Pesquisa Nacional de Saúde do Escolar 2009. Rio de Janeiro: Instituto Brasileiro de Geografia e Estatística; 2009.

11. Petroski EL, Pelegrini A. Associação entre o estilo de vida dos pais e a composição corporal dos filhos adolescentes. Rev Paul Pediatr 2009; 27:48-52.

12. Malcon MC, Menezes AMB, Chattkin M. Prevalência e fatores de risco para tabagismo em adolescentes. Rev Saúde Pública 2003; 37:1-7.

13. Hung CC, Yen LL, Wu WC. Association of parents' alcohol use and family interaction with the initiation of alcohol use by sixth graders: a preliminary study in Taiwan. BMC Public Health 2009; 9:1-9.

14. Monteiro P, Victora C, Barros F. Fatores de risco sociais, familiares e comportamentais para a obesidade em adolescentes. Rev Panam Salud Pública 2004; 16:250-7.

15. Otten R, Engels RC, Prinstein MJ. A prospective study of perception in adolescent smoking. J Adolesc Health 2009; 44:478-84.

16. Sirard JR, Laska MN, Patnode CD, Farbakhsh K, Lytle LA. Adolescent physical activity and screen time: associations with the physical home environment. Int J Behav Nutr Phys Act 2010; 7:82.
17. Campbell KJ, Crawford DA, Salmon J, Carver A, Garnett SP, Baur LA. Associations between the home food environment and obesity-promoting eating behaviors in adolescence. Obesity 2007; 15:719-30.

18. Instituto Brasileiro de Geografia e Estatística. Censo demográfico de 2010. Rio de Janeiro: Instituto Brasileiro de Geografia e Estatística; 2010.

19. Secretaria Municipal de Educação e Cultura, Prefeitura Municipal de Barão do Triunfo. Plano Municipal de Educação de Barão do Triunfo. Barão do Triunfo: Secretaria Municipal de Educação; 2009.

20. Instituto Brasileiro de Geografia e Estatística. Pesquisa Nacional por Amostra de Domicílios de 1999. Rio de Janeiro: Instituto Brasileiro de Geografia e Estatística; 2000.

21. Instituto Nacional de Estudos e Pesquisas Educacionais Anísio Teixeira. Censo escolar 2009. Brasília: Ministério da Educação; 2009.

22. Lohman TG, Roche AF, Martorell R. Anthropometric standardisation reference manual. Champaign: Human Kinetics Books; 1988.

23. Bastos JP, Araújo CLP, Hallal PC. Prevalence of insufficient physical activity and associated factors in Brazilian adolescents. J Phys Activity Health 2008; 5:777-94

24. Biddle S, Cavill N, Sallis J. Young and active? Young people and health-enhancing physical activity-evidence and implications. London: Health Education Authority; 1998.

25. Craig CL, Marshall AL, Sjöström M, Bauman AE, Booth ML, Ainsworth BE, et al. International Physical Activity Questionnaire: 12-country reliability and validity. Med Sci Sports Exerc 2003; 35:1381-95.

26. Hallal PC, Gomez LF, Parra DC, Lobelo F, Mosquera J, Florindo AA, et al. Lessons learned after 10 years of IPAQ use in Brazil and Colombia. J Phys Act Health 2010; 7 Suppl 2:S259-64.

27. Hallal PC, Victora CG, Wells JC, Lima RC. Physical inactivity: prevalence and associated variables in Brazilian adults. Med Sci Sports Exerc 2003; 35:1894-900.

28. Haskell WL, Lee IM, Pate RR, Powell KE, Blair SN, Franklin BA, et al. Physical activity and public health: updated recommendation for adults from the American College of Sports Medicine and the American Heart Association. Circulation 2007; 39:1423-34.

29. Coordenação Geral da Política de Alimentação e Nutrição, Ministério da Saúde. Alimentação saudável para todos: siga os 10 passos. Brasília: Ministério da Saúde; 2006.

30. Couto SF. Frequência de hábitos alimentares saudáveis em adolescentes do Ensino Médio de Pelotas/RS [Dissertação de Mestrado]. Pelotas: Escola Superior de Educação Física, Universidade Federal de Pelotas; 2010.

31. Carlini EA, Galduroz JCF, Noto AR, Nappo SA. I Levantamento Domiciliar sobre Drogas Psicotrópicas no Brasil. Estudo envolvendo as 107 maiores cidades do país - 2001. São Paulo: Centro Brasileiro de Informações sobre Drogas Psicotrópicas, Universidade Federal de São Paulo; 2002. 
32. Must A, Dallal GE, Dietz WH. Reference data for obesity: 85th and 95th percentiles of body mass index (wt/ht2) and triceps skinfold thickness. Am J Clin Nutr 1991; 53:839-46.

33. World Health Organization. Physical status: the use and interpretation of anthropometry: report of a WHO expert committe. Geneva: World Health Organization; 1995.

34. Barros AJ, Hirakata VN. Alternatives for logistic regression in cross-sectional studies: an empirical comparison of models that directly estimate the prevalence ratio. BMC Med Res Methodol 2003; 3:21.

35. Barreto SM, Giatti L, Casado L, Moura L, Crespo C, Malta DC. Exposição ao tabagismo entre escolares no Brasil. Ciênc Saúde Coletiva 2010; 15:3027-34.

36. Vieira PC, Aerts DRGC, Freddo SL, Bittencourt A, Monteiro L. Uso de álcool, tabaco e outras drogas por adolescentes escolares em município do Sul do Brasil. Cad Saúde Pública 2008; 24:2487-98.

37. Martínez Maldonado R, Pedrão LJ, Alonso Castillo MM, López García KS, Oliva Rodríguez NN. Selfesteem, perceived self-efficacy, consumption of tobacco and alcohol in secondary students from urban and rural areas of Monterrey, Nuevo León, México. Rev Lat Am Enfermagem 2008;16 Spec No:614-20.

38. Bernat DH, Erickson DJ, Widome R, Perry CL, Forster JL. Adolescent smoking trajectories: result from a population-based cohort study. J Adolesc Health 2008; 43:334-40.

39. Bordin R, Nipper VB, Silva JO, Bortolomiol L. Prevalência de tabagismo entre escolares em município de área metropolitana da Região Sul, Brasil, 1991. Cad Saúde Pública 1993; 9:185-9.

40. Precioso J, Macedo M, Rebelo L. Relação entre o tabagismo dos pais e o consumo de tabaco dos filhos: implicações para a prevenção. Revista Portuguesa de Clínica Geral 2007; 23:259-66.

41. Muza GM, Costa MP. Aspectos sociofamiliares do consumo de tabaco por adolescentes escolares da rede privada do Distrito Federal. Revista ABP-APAL 1993; 15:31-6.

42. Rudatsikira E, Muula AS, Siziya S, Mataya RH. Correlates of cigarette smoking among school-going adolescents in Thailand: findings from the Thai global youth tobacco survey 2005. Int Arch Med 2008; 1:8.

43. Malta DC, Sardinha LMV, Mendes I, Barreto SM, Giatti L, Castro IRR, et al. Prevalência de fatores de risco e proteção de doenças crônicas não transmissíveis em adolescentes: resultados da Pesquisa Nacional de Saúde do Escolar (PeNSE), Brasil, 2009. Ciênc Saúde Coletiva 2010; 15:3009-19.

44. Strauch ES, Pinheiro RT, Silva RA, Horta BL. Uso de álcool por adolescentes: estudo de base populacional. Rev Saúde Pública 2009; 43:647-55.

45. Tavares BF, Béria JU, Lima MS. Fatores associados ao uso de drogas entre adolescentes escolares. Rev Saúde Pública 2004; 38:787-96.

46. Alavarse GMA, Carvalho MDB. Álcool e adolescência: o perfil de consumidores de um município do norte do Paraná. Esc Anna Nery Rev Enferm 2006; 10:408-16.
47. Noal RB, Menezes AMB, Araujo CL, Hallal PC. Experimental use of alcohol in early adolescence: the 11-year follow-up of the 1993 Pelotas (Brazil) birth cohort study. Cad Saúde Pública 2010; 26:1937-44.

48. Rojas-Guiot E, Fleiz-Bautista C, Medina-Mora Icaza ME, Morón MA, Domenech-Rodríguez M. Consumo de alcohol y drogas en estudiantes de Pachuca, Hidalgo. Salud Pública Méx 1999; 41:297-308.

49. Vieira DL, Ribeiro M, Romano M, Laranjeira RR. Álcool e adolescentes: estudo para implementar políticas municipais. Rev Saúde Pública 2007; 41:396-403.

50. Guillén RR, Nascimento LC. Consumo de drogas en los jóvenes de la ciudad de Guayaquil, Ecuador. Rev Lat Am Enfermagem 2010; 18 (Spec):598-605.

51. Recine E, Radaelli P. Alimentação e cultura. Brasília: Ministério da Saúde; 2008. http://bvsms.saude. gov.br/bvs/publicacoes/alimentacao_cultura.pdf (acessado em 12/Mai/2011).

52. Ogden CL, Carroll MD, Curtin LR, McDowell MA, Tabak CJ, Flegal KM. Prevalence of overweight and obesity in the United States, 1999-2004. JAMA 2006; 295:1549-55.

53. Bonvecchio A, Safdie M, Monterrubio EA, Gust T, Villalpando S, Rivera JA. Overweight and obesity trends in Mexican children 2 to 18 years of age from 1988 to 2006. Salud Pública Méx 2009; 51 Suppl 4:S586-94.

54. Magalhães VC, Mendonça GAS. Prevalência e fatores associados a sobrepeso e obesidade em adolescentes de 15 a 19 anos das regiões Nordeste e Sudeste do Brasil, 1996 a 1997. Cad Saúde Pública 2003; 19 Suppl 1:S129-39.

55. Silva GAP, Balaban G, Motta MEF. Prevalência de sobrepeso e obesidade em crianças e adolescentes de diferentes condições socioeconômicas. Rev Bras Saúde Matern Infant 2005; 5:53-9.

56. Campos LA, Leite AJM, Almeida PC. Nível socioeconômico e sua influência sobre a prevalência de sobrepeso e obesidade em escolares adolescentes do município de Fortaleza. Rev Nutr 2006; 19:531-3.

57. Delwing KBB, Rempel C, Bosco SMD. Prevalência de sobrepeso e obesidade em escolares entre 6 e 11 anos de um município do interior do RS. ConScientiae Saúde 2010; 9:173-8.

58. Amaral APA, Palma, AP. Perfil epidemiológico da obesidade em crianças: relação entre televisão, atividade física e obesidade. Rev Bras Ciênc Mov 2001; 4:19-24.

59. Clark HR, Goyder E, Bissell P, Blank L, Peters J. How do parents' child-feeding behaviours influence child weight? Implications for childhood obesity policy. J Public Health (Oxf.) 2007; 29:132-41.

60. Silva ARV, Damasceno MMC, Marinho NBP, Almeida LS, Araújo MFM, Almeida PC, et al. Hábitos alimentares de adolescentes de escolas públicas de Fortaleza, CE, Brasil. Rev Bras Enferm 2009; 62:18-24.

61. Neutzling MB, Araújo CL, Vieira MFA, Hallal PC, Menezes AMB, Victora CG. Intake of fat and fiberrich foods according to socioeconomic status: the 11-year follow-up of the 1993 Pelotas (Brazil) birth cohort study. Cad Saúde Pública 2010; 26:1904-11. 
62. Ortiz-Hernandez L, Gomez-Tello BL. Food consumption in Mexican adolescents. Rev Panam Salud Pública 2008; 24:127-35.

63. Rasmussen M, Krølner R, Klepp K-I, Lytle L, Brug J, Bere E, et al. Determinants of fruit and vegetable consumption among children and adolescents: a review of the literature. Part I: quantitative studies. Int J Behav Nutr Phys Act 2006; 3:22.

64. Levy RB, Castro IRR, Cardoso LO, Tavares LF, Sardinha LMV, Gomes FS, et al. Consumo e comportamento alimentar entre adolescentes brasileiros. Pesquisa Nacional de Saúde do Escolar (PeNSE), 2009. Ciênc Saúde Coletiva 2010; 15 Suppl 2:S3085-97.

65. Vitolo MR, Campagnolo PDB, Gama CM. Factors associated with risk of low dietary fiber intake in adolescents. J Pediatr 2007; 83:47-52.

66. Pearson N, Timperio A, Salmon J, Crawford D, Biddle SJH. Family influences on children's physical activity and fruit and vegetable consumption. Int J Behav Nutr Phys Act 2009; 6:1-7.

67. Monge-Rojas R, Garita C, Sánchez M, Muñoz L. Barriers and motivators for healthful eating as perceived by rural and urban Costa Rican adolescents. J Nutr Educ Behav 2005; 37:33-40.

68. Reichert FF, Menezes AMB, Araújo CL, Hallal PC. Self-reporting versus parental reporting of physical activity in adolescents: the 11-year follow-up of the 1993 Pelotas (Brazil) birth cohort study. Cad Saúde Pública 2010; 26:1921-7.
69. Hallal PC, Knuth AG, Cruz DKA, Mendes MI, Malta DC. Prática de atividade física em adolescentes brasileiros. Ciênc Saúde Coletiva 2010; 15 Suppl 2:S3035-42.

70. Tenório MCM, Barros MVG, Tassitano RM, Bezerra J, Hallal PC. Atividade física e comportamento sedentário em adolescentes estudantes do Ensino Médio. Rev Bras Epidemiol 2010; 13:105-17.

71. Richards R, Poulton R, Reeder AI, Williams S. Childhood and contemporaneous correlates of adolescent leisure time physical inactivity: a longitudinal study. J Adolesc Health 2009; 44:260-7.

72. Gonçalves H, Hallal PC, Amorim TC, Araújo CL, Menezes AM. Fatores socioculturais e nível de atividade física no início da adolescência. Rev Panam Salud Pública 2007; 22:246-53.

73. Anderssen N, Wold B, Torsheim T. Are parental health habits transmitted to their children? An eight-year longitudinal study of physical activity in adolescents and their parents. J Adolesc 2006; 29:513-24.

74. Dumith SC, Domingues MR, Gigante DP. Estágios de mudança de comportamento para a prática de atividade física: uma revisão da literatura. Rev Bras Cineantropom Desempenho Hum 2008; 10:301-7.

Recebido em 16/Abr/2011

Versão final reapresentada em $27 / \mathrm{Jul} / 2011$

Aprovado em 22/Ago/2011 